\title{
Pancreatoduodenectomy for groove pancreatitis: A case report and literature review
}

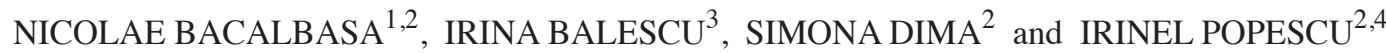 \\ ${ }^{1}$ Department of Obstetrics and Gynecology, 'Carol Davila' University of Medicine and Pharmacy, 020021 Bucharest; \\ ${ }^{2}$ Department of Visceral Surgery, Center of Excellence in Translational Medicine, 'Fundeni' Clinical Institute, \\ 022328 Bucharest; ${ }^{3}$ Department of Visceral Surgery, 'Ponderas' Academic Hospital, 021188 Bucharest; \\ ${ }^{4}$ Department of Visceral Surgery, 'Titu Maiorescu' University of Medicine and Pharmacy, 040051 Bucharest, Romania
}

Received July 26, 2021; Accepted August 25, 2021

DOI: $10.3892 / \mathrm{etm} .2021 .10890$

\begin{abstract}
Groove pancreatitis represents a rare pathological condition which is usually associated with chronic alcohol intake. However, the differential diagnosis between groove pancreatitis and groove pancreatic carcinoma is difficult to establish pre-operatively. In this respect, a significant number of cases are treated as malignant conditions, with the final diagnosis of benign disease being established post-operatively. The present study describes the case of a 46-year-old male who was diagnosed with groove pancreatitis. Due to the uncertainty of the pre-operative diagnosis, the patient was subjected to radical surgery consisting of pancreatoduodenectomy. The final histopathological diagnosis confirmed the presence of groove pancreatitis. Moreover, it excluded the possibility of an association with any element of malignancy and provided the effective management of the disease, all the symptoms being significantly alleviated at the 3-month follow-up. In addition, the present study also provides a brief summary and discussion of the disease and associated treatment options. On the whole, as demonstrated herein, pancreatoduodenectomy appears to be a safe and effective method for the treatment of groove pancreatitis, being associated with a significant improvement in the quality of life of the patient; moreover, it represents the sole method which can exclude the diagnosis of malignancy.
\end{abstract}

Correspondence to: Dr Nicolae Bacalbasa, Department of Obstetrics and Gynecology, 'Carol Davila' University of Medicine and Pharmacy, Bulevardul Eroilor Sanitari 8, 020021 Bucharest, Romania

E-mail: nicolae_bacalbasa@yahoo.ro

Key words: groove pancreatitis, pancreatoduodenectomy, symptoms, weight gain, differential diagnostic, histopathological result

\section{Introduction}

Also known as paraduodenal pancreatitis, paraduodenal wall cyst, myoadenomatosis or cystic dystrophy, groove pancreatitis represents a distinct form of inflammation of the pancreatic groove and was first described by Becker in 1973 (1,2). The groove area refers to the narrow space located between the pancreatic head medially, the duodenum II laterally, the duodenum III and inferior vena cava posteriorly and the duodenum I superiorly (3). According to the study by Becker and Mischke (2), groove pancreatitis can present as pure-type or as segmental-type. One of the most significant issues which should be taken into consideration in cases in which groove pancreatitis is suspected is related to the differential diagnosis with groove pancreatic carcinoma (4-7). Due to the extremely low number of such cases, the pre-operative diagnosis is difficult to establish. In this respect, the majority of cases are subjected to surgery with a pre-operative suspicion of malignant disease, with the final diagnosis being established upon a histopathological examination. Therefore, in such cases, pancreatoduodenectomy has become the treatment of choice $(4,5)$.

\section{Case report}

The present study describes the case of a 46-year-old male, with a previous history of alcoholic pancreatitis, who was submitted to surgery for pre-operative diagnosis of pancreatic cancer. The data of the patient were retrospectively reviewed after obtaining the approval of the Ethics Committee of 'Fundeni' Clinical Institute (approval no. 267/2020). Patient consent for publication was obtained and signed by the patient on March 19, 2020.

The 46-year-old patient with a known history of heavy alcoholic intake and several episodes of acute pancreatitis was self-referred to the 'Fundeni' Clinical Institute (Bucharest, Romania) for weight loss, abdominal pain, nausea, vomiting and a weight loss of $12 \mathrm{~kg}$ in the last 3 months. A computed tomography scan revealed the presence of a hypodense mass at the level of the pancreatic head, measuring 4/5/3 cm apparently with no vascular invasion (Figs. 1 and 2).

The patient was further subjected to an endoscopic ultrasound and biopsy examination, which revealed the 


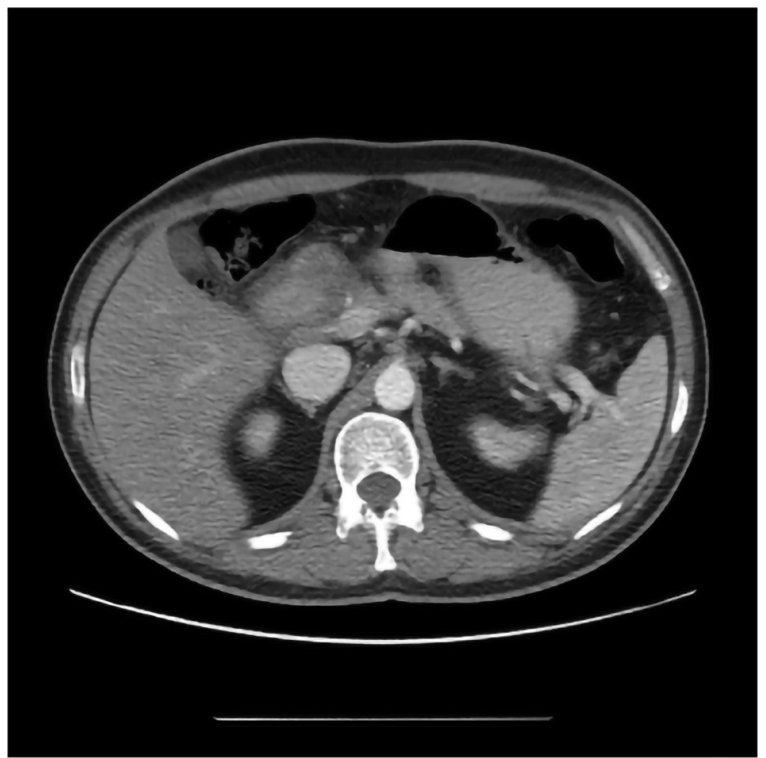

Figure 1. Computed tomography scan revealing the presence of a hypodense mass located at the level of the pancreatic head.

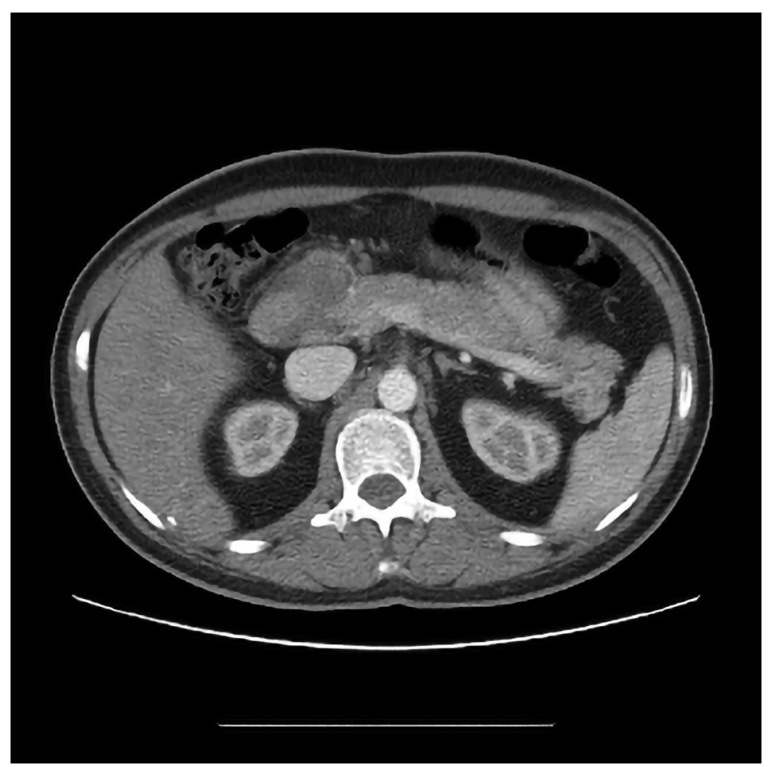

Figure 2. Computed tomography revealing low-density area in the early phase due to a large-fiber fibrosis component.

presence of chronic inflammation, as well as the absence of malignant cells. However, the biological test results revealed an increase in the levels of serum markers when compared to the determination which had been performed 6 months prior; at this time, the serum level of CA 19-9 was $78 \mathrm{U} / \mathrm{ml}$, whereas 6 months prior, these levels were $33 \mathrm{U} / \mathrm{ml}$. Thus, the patient was submitted to surgery for a pancreatoduodenectomy (Figs. 3 and 4).

The post-operative outcome was uneventful, with the patient being discharged on day 10 post-operatively. However, the histopathological examination confirmed the absence of malignant cells in the specimen, and the finally diagnosis of groove pancreatitis was made. At the 3-month follow-up period, the patient reported a significant improvement in his

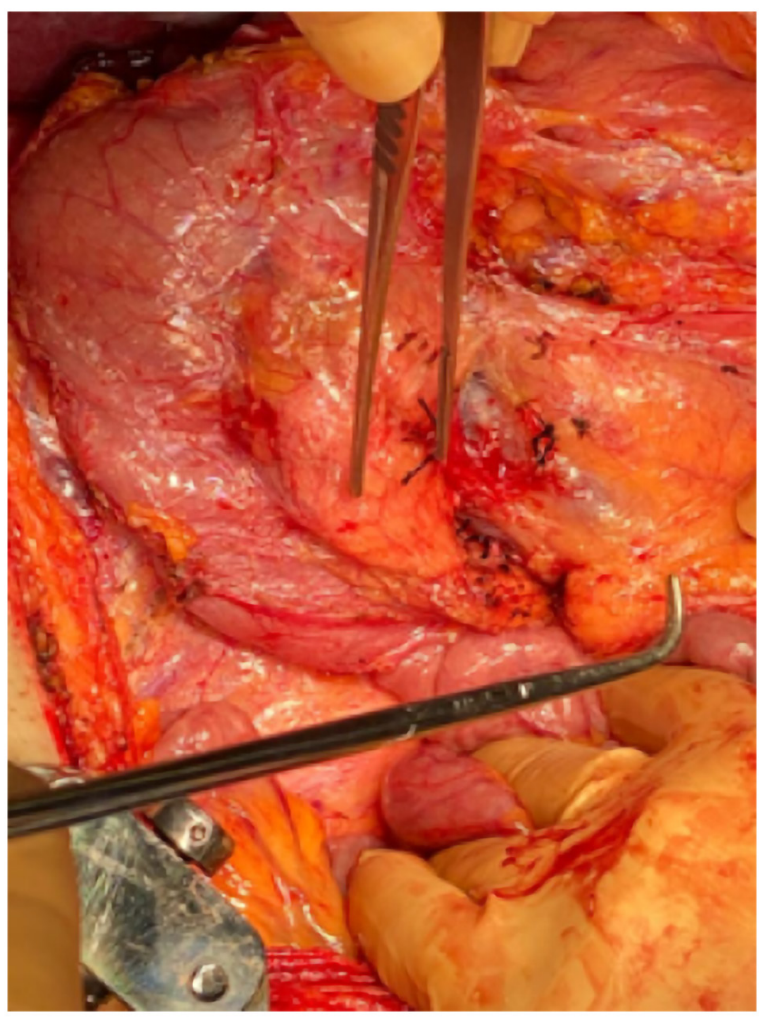

Figure 3. Intraoperative aspect following the dissection of the superior mesenteric vessels.

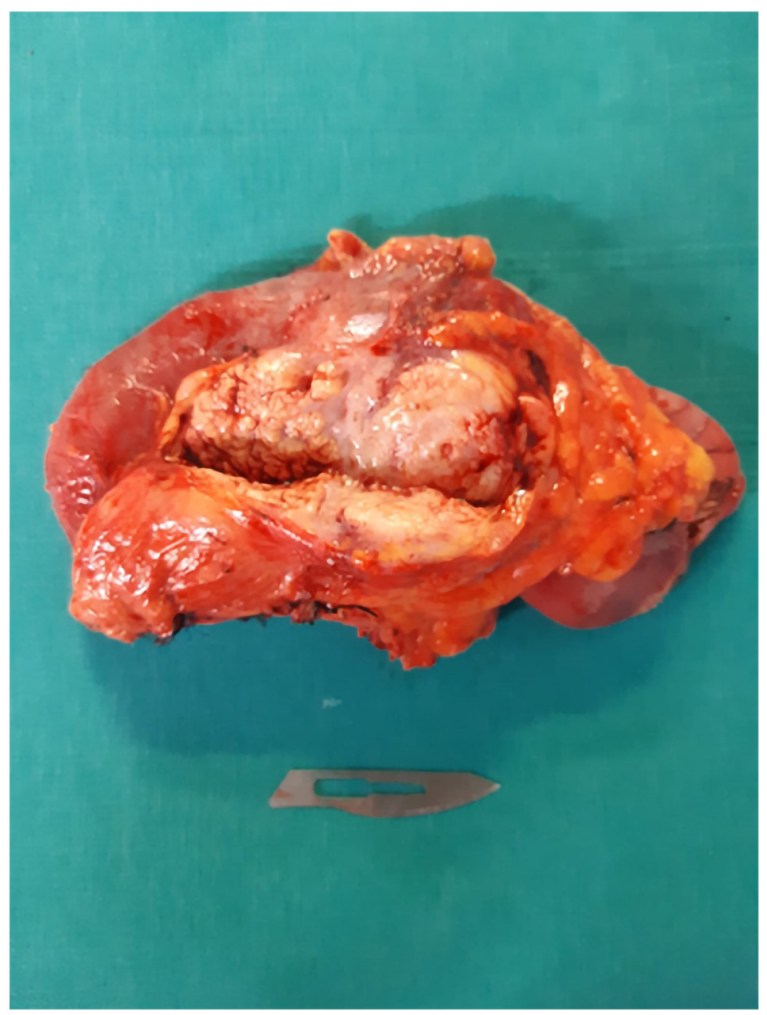

Figure 4. Final aspect of the specimen: Fibrotic and inflammatory aspect of the pancreatic groove.

clinical status, with the alleviation of abdominal pain, as well as a weight gain of $4.5 \mathrm{~kg}$. 


\section{Discussion}

Initially described by Becker in 1973 (1), groove pancreatitis was classified by Stolte et al (7) as having two forms: A pure one, which is characterized by the presence of inflammatory and fibrous lesions affecting exclusively the virtual space between the pancreatic head and the duodenum, and a segmental form affecting the pancreatic head medially. In the latter situation, the surrounding structures, such as the common biliary duct, duct of Wirsung or duodenum are progressively involved, leading to the development of jaundice, abdominal pain, weight loss or post-prandial vomiting $(8,9)$. Mainly associated with chronic alcohol abuse, groove pancreatitis has been also described in patients with duodenal peptic ulcers or congenitally in children (3).

As regards the types of therapeutic strategies, patients diagnosed with groove pancreatitis can be initially subjected to medical therapies in order to provide an effective analgesic effect and to provide an adequate substitution of pancreatic function $(10,11)$. In cases in which the symptoms are solely produced by pancreatic duct obstruction, endoscopic procedures in order to provide an effective drainage of the pancreatic secretion via endoscopy may be taken in consideration (12). However, this method may not be applicable in cases presenting with severe duodenal stenosis or in which the pre-operative investigations could not exclude the association of malignant transformation (3). In cases in which conservative treatment fails and symptoms recur, surgery should be taken in consideration; in this respect, various procedures have been proposed, ranging from duodenal and biliary bypass, duodenum preserving pancreatic head resection or pancreatoduodenectomy $(13,14)$; however, conservative procedures, such as bypass or segmental resections have been more commonly proposed in pediatric patients, cases in which the diagnosis of malignancy is not probable (3). Moreover, the necessity of resecting large malignant tumors alone or in association with vascular resections have led to the widespread use of pancreatoduodenectomy as the primary therapeutic option for patients with groove pancreatitis (15-18).

One of the first studies which demonstrated the difficulties in differentiating groove pancreatic cancer from groove pancreatitis was conducted by Yamaguchi and Tanaka (19) and was published in 1992. The study included 8 patients from six institutions; in all the patients, a pre-operative diagnosis could not be established. The main features which made the diagnosis of malignant disease a very probable one were represented by the presence of extensive duodenal stenosis, common bile duct stenosis or vascular encasement. In all cases, a pancreatoduodenectomy was performed and the histopathological analyses demonstrated the absence of malignant disease (19).

One of the largest single institutional studies conducted on groove pancreatitis was published in 2007 by Rahman et al (20); the study included 11 patients investigated for abdominal pain, gastric outlet obstruction or jaundice. In all cases, pancreatoduodenectomy was performed, with the analysis of the specimens demonstrating the absence of malignant transformation; moreover, following a median follow-up period of 52 weeks, all patients reported weight gain, with a mean value of $3 \mathrm{~kg}$ at 2 months of follow-up, as well as a significant alleviation of pain, demonstrating in this manner, the effectiveness of resection in such cases (19). The benefit in terms of the alleviation of abdominal pain was explained by the interruption of the local fibrotic and inflammatory networks $(20,21)$.

The widespread demonstration of the benefits of pancreatoduodenectomy in the field of groove pancreatitis has led to the inclusion of this procedure as standard of care in the latest guidelines. Therefore, in the United European Gastroenterology evidence-based guidelines for the diagnostic and therapy of chronic pancreatitis, published in 2017, the interviewed experts declared that in groove pancreatitis, pancreatoduodenectomy represents the most suitable surgical option (the statement being considered as strongly agreeable) (21).

In conclusion, groove pancreatitis represents a rare entity, with only isolated cases being reported thus far; moreover, the differential diagnosis with pancreatic groove cancer is usually difficult to establish pre-operatively. Therefore, in the majority of cases, the treatment of choice remains pancreatoduodenectomy, with the final diagnosis being established at the time of the histopathological examination. Furthermore, performing a wide resection of the lesion seems to have an overall positive influence on the quality of life of patients, with a significant alleviation of pain, as well as weight gain being reported in the ensuing months.

\section{Acknowledgements}

Not applicable.

\section{Funding}

No funding was received.

\section{Availability of data and materials}

The datasets used and/or analyzed during the current study are available from the corresponding author on reasonable request.

\section{Authors' contributions}

NB contributed to the conception of the study, collected, analyzed and interpreted data from the literature and the data corresponding to the patient, and critically revised the manuscript. IB contributed to the conception of the study, performed the literature research and drafted the manuscript. SD contributed to the conception of the study, performed the literature research and drafted the manuscript. IP collected, analyzed and interpreted the data corresponding to the patient and critically revised the manuscript. IB and SD confirm the authenticity of all the raw data. All authors have read and approved the final manuscript.

\section{Ethics approval and consent to participate}

The Ethics Committee of 'Fundeni' Clinical Institute approved the study.

\section{Patient consent for publication}

Patient consent for publication was obtained and signed by the patient on March 19, 2020. 


\section{Competing interests}

The authors declare that they have no competing interests.

\section{References}

1. Becker V: Bauchspeicheldrüse. In: Spezielle pathologische Anatomie. Doerr W, Seifert G and Uhlinger E (eds). Vol 4. Springer, Berlin, 1973 (In German).

2. Becker V and Mischke U: Groove pancreatitis. Int J Pancreatol 10: 173-182, 1991

3. Tahara K, Kanamor Y, Miyake K, Kudo Y,Fujita T, Kutsukake M, Mori T, Yamada Y, Fujino A, Shimizu H, et al: Groove pancreatitis treated by duodenal and biliary bypass. J Pediatr Surg Case Rep 59: 101540, 2020.

4. Yabuki K, Maekawa T, Satoh K, Tamasaki Y, Maekawa H, Amano T, Ogawa K and Anouma K: A case of groove pancreatitis with duodenal stenosis. Dig Endosc 12: 345-349, 2000.

5. Munthali Lovemore CE, Hsu JT, Chiu CT, Chen HM and Chen MF: Groove pancreatitis: Case report and literature review. Chang Gung Med J 24: 512-516, 2001.

6. Dey R and Mannion R: Groove pancreatitis-A case report and review of imaging features. Eur J Radiol Extra 64: 67-69, 2007.

7. Stolte M, Weiss W, Volkholz H and Rosch W: A special form of segmental pancreatitis: 'Groove Pancreatitis' Hepatogastroenterology 29: 198-208, 1982.

8. Manzelli A, Petrou A, Lazzaro A, Brennan N, Soonawalla Z and Friend P: Groove pancreatitis. A mini-series report and review of the literature. JOP 12: 230-233, 2011.

9. Frutos-Perez JM, Perea-Ribis M, Martinez-Pascual MA Llopis-Sanchis M and Tornero-Estebanez C: Constitutional syndrome, ascites and duodenal thickening presenting as groove pancreatitis. Eur J Case Rep Intern Med 5: 000789, 2018.

10. Aguilera F, Tsamalaidze L, Raimondo M, Puri R, Asbun HJ and Stauffer JA: Pancreaticoduodenectomy and outcomes for groove pancreatitis. Dig Surg 35: 475-481, 2018.

11. Balduzzi A, Marchegiani G, Andrianello S, Romeo F, Amodio A, De Pretis N, Zamboni G, Malleo G, Frulloni L, Salvia R and Bassi C: Pancreaticoduodenectomy for paraduodenal pancreatitis is associated with a higher incidence of diabetes but a similar quality of life and pain control when compared to medical treatment. Pancreatology 20: 193-198, 2020.
12. Chantarojanasiri $\mathrm{T}$, Isayama $\mathrm{H}$, Nakai $\mathrm{Y}$, Matsubara $\mathrm{S}$, Yamamoto N, Takahara N, Mizuno S, Hamada T, Kogure H and Koike K: Groove pancreatitis: Endoscopic treatment via the minor papilla and duct of santorini morphology. Gut Liver 12: 208-213, 2018.

13. Zhu C, Huang Q, Zhu J, Zhang X and Qin X: Groove resection of pancreatic head in groove pancreatitis: A case report. Exp Ther Med 14: 1983-1988, 2017.

14. Tezuka K, Makino T, Hirai I and Kimura W: Groove pancreatitis. Dig Surg 27: 149-152, 2010.

15. Brasoveanu V, Anghel C, Barbu I, Pautov M, Ionescu MI, Motthor M, Balescu I, Dima S and Bacalbasa N: Pancreatoduodenectomy en bloc with portal and superior mesenteric artery resection-a case report and literature review. Anticancer Res 35: 1613-1618, 2015.

16. Bacalbasa N, Balescu I, Dima S, Brasoveanu V and Popescu I: Pancreatic resection as part of cytoreductive surgery in advanced-stage and recurrent epithelial ovarian cancer-A single-center experience. Anticancer Res 35: 4125-4129, 2015.

17. Brasoveanu V, Dumitrascu T, Bacalbasa N and Zamfir R: Splenic artery used for replaced common hepatic artery reconstruction during pancreatoduodenectomy-a case report. Chirurgia (Bucur) 104: 499-504, 2009.

18. Bacalbasa N, Balescu I, Tanase A, Pautov M, Brezean I, Vilcu M and Brasoveanu V: Spleno-Pancreatectomy en bloc with parcelar gastrectomy for splenic artery aneurysm-a case report and literature review. In Vivo 32: 915-919, 2018.

19. Yamaguchi K and Tanaka M: Groove pancreatitis masquerading as pancreatic carcinoma. Am J Surg 163: 312-316, 1992.

20. Rahman SH, Verbeke CS, Gomez D, McMahon MJ and Menon KV: Pancreatico-duodenectomy for complicated groove pancreatitis. HPB (Oxford) 9: 229-234, 2007.

21. Löhr JM, Dominguez-Munoz E, Rosendahl J, Besselink M, Mayerle J, Lerch MM, Haas S, Akisik F, Kartalis N, Iglesias-Garcia J, et al: United European gastroenterology evidence-based guidelines for the diagnosis and therapy of chronic pancreatitis (HaPanEU). United European Gastroenterol J 5: 153-199, 2017. 